\section{JTI}

JOURNAL OF

TRAUMA AND INJURY

\title{
Non-Permanent Transcatheter Proximal Renal Artery Embolization for a Grade 5 Renal Injury with Delayed Recanalization and Preserved Renal Parenchymal Enhancement
}

\author{
Abhishek Jairam, M.D., Bradley King, B.A., Zachary Berman, M.D., \\ Gerant Rivera-Sanfeliz, M.D. \\ Department of Radiology, University of California, San Diego, La Jolla, CA, USA
}

Revised: February 25, 202

Accepted: May 31, 2021

\section{Correspondence to}

Abhishek Jairam, M.D.

Department of Radiology, University of California, San Diego, 9500 Gilman Dr.

La Jolla, CA 92093, USA

Tel: +1-805-796-7299

E-mail:abjairam@health.ucsd.edu ORCID: https://orcid.org/0000-00027374-6407

\begin{abstract}
Super-selective renal artery embolization is an increasingly popular technique for the management of traumatic, low-grade renal trauma. When performed in distal arterial branches, this intervention enables tissue preservation and arrest of hemorrhage, but it may not be practical in cases of multifocal, high-grade renal injuries. In such cases, surgical nephrectomy remains the more common treatment modality to ensure hemodynamic control. We present the unique case of a patient who presented in hemorrhagic shock following a major trauma that resulted in a grade 5 renal injury treated with complete renal artery embolization using Gelfoam, resulting in hemodynamic stabilization. Interestingly, imaging 1 month after embolization revealed residual enhancement of the inferior pole of the kidney, suggesting reconstitution of flow and partial renal salvage. Ultimately, transcatheter "nephrectomy" with careful selection of a temporary embolic agent may serve as a safe and efficient alternative to surgical nephrectomy with the added possibility of preserving partial renal perfusion and function in the emergent setting.
\end{abstract}

Keywords: Renal trauma; Nephrectomy; Embolization; Gel foam

\section{INTRODUCTION}

Renal injuries occur in $1-5 \%$ of patients with trauma and are graded according to the American Association for the Surgery of Trauma classification system [1]. The most (http://creativecommons.org/licenses/by-nc/4.0/) which permits unrestricted noncommercial use, distribution, and reproduction in any medium, provided the original work is properly cited. 
severe form of renal injury (grade $\mathrm{V}$ ) is characterized by a shattered kidney, avulsion of the renal hilum, or complete laceration or thrombosis of the main renal artery or vein, leading to devascularization of the kidney. Traditionally, surgical management is performed for high-grade renal injuries, with nephrectomy rates as high as $83 \%$ [2]. Transarterial embolization of the lobar and second-/third-order branches of the renal artery is an increasingly popular, tissue-preserving alternative, with studies demonstrating safe arrest of hemorrhage and minimal impact on renal function [3-5]. Although repeat embolization is often required after the initial intervention for grade IV and $\mathrm{V}$ injuries, the renal salvage rate remains robust at nearly $90 \%$ [6].

Careful consideration of both the type of embolic agent and level of renal arterial injury plays an important role in dictating safe and efficient treatment in renal trauma. In cases of second- or third-order renal arterial injuries, super-selective coil embolization as distal as possible remains a common approach to control bleeding and preserve renal parenchyma [7]. However, in cases of multifocal renal injury, this approach may be technically prohibitive and require a longer procedure time than is feasible for an unstable patient. In such cases, the best non-surgical treatment option may be complete proximal renal embolization, using non-permanent embolic agents with the goal of achieving hemostasis.

We report a case of a shattered kidney successfully treated with transcatheter nephrectomy using temporary porcine gelatin slurry embolization with partial preservation of renal parenchymal enhancement in a patient with a traumatic grade 5 renal injury.

\section{CASE REPORT}

A 64-year-old male with a history of hepatitis $\mathrm{C}$ and polysubstance abuse was brought to the hospital by emergency medical services after being struck by a high-speed car while riding his bicycle. The patient experienced a temporary loss of consciousness at the scene and presented to the trauma bay in hemorrhagic shock. Resuscitation with one unit of packed red blood cells, one unit of fresh frozen plasma, and 3.0 L of crystalloid was unsuccessful in normalizing the patient's blood pressure. A pelvic X-ray revealed an open-book pelvic fracture, and a Focused Assessment with Sonography in Trauma examination was positive for free abdominal fluid concentrated in Morrison's pouch.

After providing informed consent, the patient subsequently underwent emergent exploratory laparotomy. Approximately $300 \mathrm{~mL}$ of blood originating from the pelvis was drained. A large, right-sided, non-expanding perinephric hematoma was noted, as was contusion of hepatic segment 6 . A wound vacuum was placed for temporary abdominal closure, and the patient underwent computed tomography angiography of the abdomen, revealing highgrade laceration of the right kidney and active arterial extravasation with a surrounding perirenal hematoma (Fig. 1).

The patient remained hemodynamically unstable postoperatively. Interventional radiology was ultimately consulted for emergent renal angiography for a diagnostic and therapeutic intervention. On angiography, multiple renal artery pseudoaneurysms were noted along with an accessory right renal artery (Fig. 2). Due to the patient's hemodynamic instability, temporary embolization of the main right and accessory right renal arteries was performed (Fig. 3). A completion angiogram showed occlusion of arterial inflow and hemostasis in the entirety of the kidney. Following embolization, the patient's blood pressure normalized and he did not require further resuscitation. Follow-up abdominal and pelvic computed

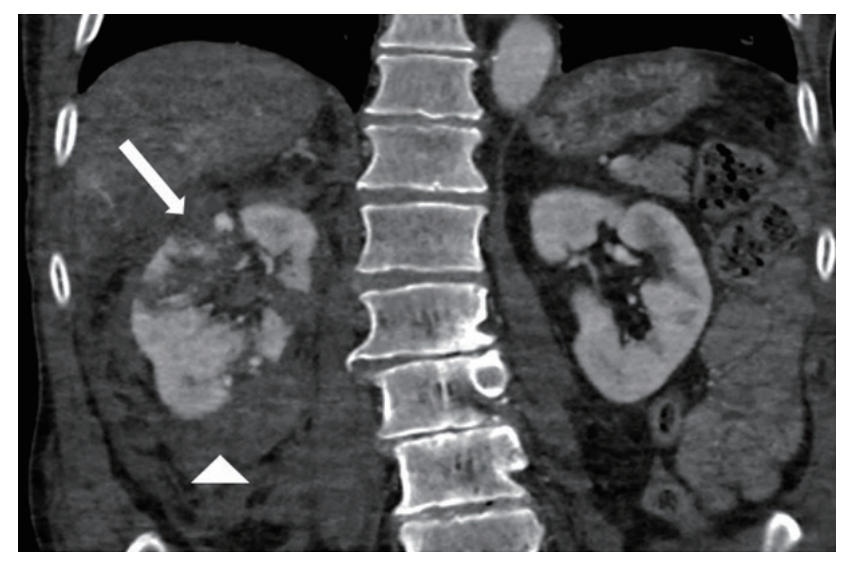

Fig. 1. Coronal contrast-enhanced computed tomography image demonstrating a right shattered kidney with surrounding perirenal hematoma (arrowhead) and complete laceration of the superior and interpolar regions (arrow). 


\section{JTI}

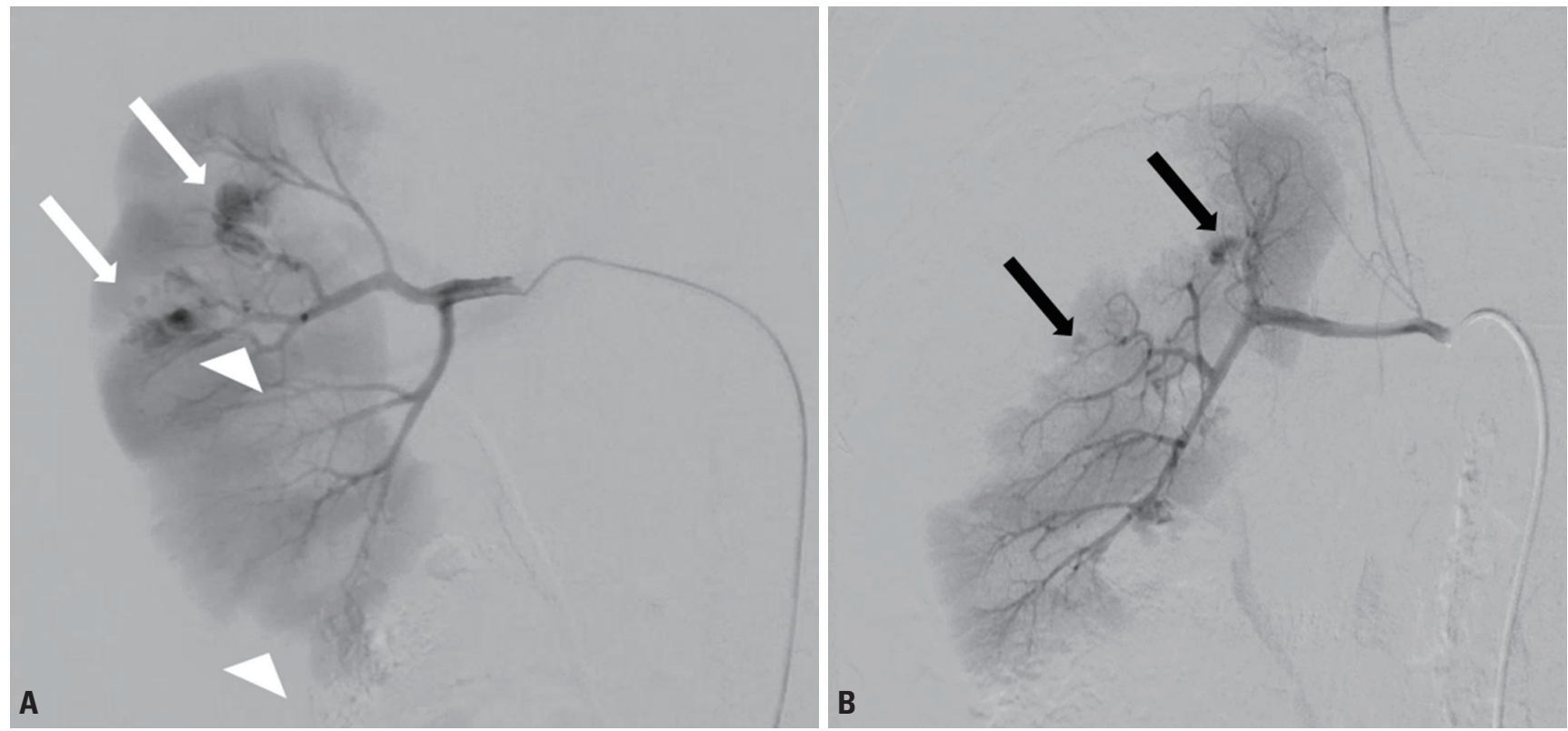

Fig. 2. (A) Anteroposterior digital subtraction arteriogram of the main right renal artery showing multifocal pseudoaneurysms (white arrows) and incomplete cortical enhancement (white arrowheads). (B) Catheterization of the accessory right renal artery with digital subtraction angiography demonstrating multiple pseudoaneurysms (black arrows) and incomplete parenchymal enhancement of the peripheral interpolar and superior polar cortices.



Fig. 3. (A) Post-Gelfoam embolization of the main (arrow) and (B) accessory (arrowhead) renal arteries demonstrating hemostatic control, resolution of pseudoaneurysms, and complete infarction as intended. 


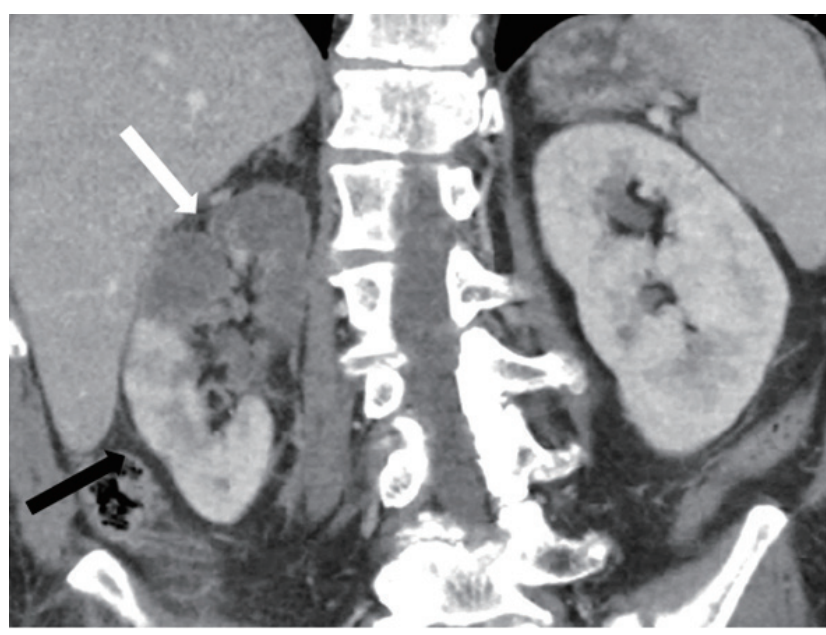

Fig. 4. Four weeks post-embolization. Coronal contrast-enhanced computed tomography image demonstrating preserved perfusion to the inferior pole of the right kidney (black arrow), with the expected evolving infarction of the superior pole (white arrow).

tomography (CT) at 2 and 4 weeks revealed an evolving infarct of the superior pole of the right kidney with preserved perfusion to the lower pole (Fig. 4).

Prior to his injury, the patient had a baseline creatinine of $0.6-0.8 \mathrm{mg} / \mathrm{dL}$ with no known renal disease. In the immediate aftermath of his hemorrhagic shock and renal embolization, he experienced a peak creatinine level of $1.39 \mathrm{mg} / \mathrm{dL}$. This value subsequently trended downward over the remainder of his hospitalization, ultimately returning to baseline, consistent with hyperfunctioning of the non-injured kidney. Diagnostic tests, including CT urography and a renal scan, were not performed due to adequate urine output and stable creatinine levels.

\section{DISCUSSION}

Traditionally, surgical nephrectomy has been the most commonly performed intervention for grade 5 renal injuries, especially for patients with hemodynamic instability. Transcatheter embolization remains an understudied therapeutic option in this population, as it is typically reserved for lower-grade injuries [7]. Furthermore, these lower-grade injuries tend to be more focal in location, making it possible for selective coil embolization to preserve renal parenchyma. In the unique case presented herein, complete renal embolization rather than surgical nephrectomy was performed in an unstable patient with a multifocal grade 5 renal injury, resulting in hemodynamic control. Partially preserved parenchymal enhancement of the inferior pole was observed on follow-up CT, suggesting viability of this tissue despite angiographically confirmed infarction of the entire kidney.

The return of renal perfusion to the inferior pole of the kidney may have been due to the use of a temporary embolic agent such as Gelfoam. In the setting of transcatheter complete renal artery embolization, the mechanism of Gelfoam is advantageous for multiple reasons. First, it results in rapid hemostasis by causing both mechanical obstruction and thrombus formation, which is particularly useful in the emergent trauma setting [8]. Second, given the temporary nature of this embolic agent, vessel recanalization is typically seen between 2 weeks to 4 months post-procedurally. In this unique case, recanalization of a second-order branch of the main renal artery likely occurred soon after the procedure, allowing for partial preservation of renal perfusion and function of the inferior pole. Small vessel collateralization to the inferior renal pole may have also contributed to the preservation of renal perfusion observed in this patient. A renal scan and CT urography can be utilized to more accurately assess whether renal perfusion has returned if a further evaluation is desired.

Ultimately, transcatheter complete renal artery embolization can be considered in the management of renal trauma with careful selection of a temporary embolic agent. This method may serve as a safe and efficient alternative to surgical nephrectomy with the added possibility of preserving renal perfusion and function in portions of the non-injured kidney.

\section{Main points}

This report demonstrates the safety and efficacy of whole-kidney embolization for the management of severe renal trauma and hemorrhage. The use of a temporary embolic agent may allow the reconstitution of flow following adequate hemostasis. Validation through a larger case series or prospective studies is warranted to compare this technique with surgical nephrectomy and to assess the complication rate. 


\section{REFERENCES}

1. Ierardi AM, Floridi C, Fontana F, Duka E, Pinto A, Petrillo M, et al. Transcatheter embolisation of iatrogenic renal vascular injuries. Radiol Med 2014;119:261-8.

2. Wessells H, Suh D, Porter JR, Rivara F, MacKenzie EJ, Jurkovich GJ, et al. Renal injury and operative management in the United States: results of a population-based study. J Trauma 2003;54:423-30.

3. Sarani B, Powell E, Taddeo J, Carr B, Patel A, Seamon M, et al. Contemporary comparison of surgical and interventional arteriography management of blunt renal injury. J Vasc Interv Radiol 2011;22:723-8.

4. Brewer ME Jr, Strnad BT, Daley BJ, Currier RP, Klein FA, Mobley JD, et al. Percutaneous embolization for the management of grade 5 renal trauma in hemodynamically unstable patients: initial experience. J Urol 2009;181:1737-41.

5. amaswamy RS, Akinwande O, Tiwari T. Renal embolization: current recommendations and rationale for clinical practice. Curr Urol Rep 2018;19:5.

6. Hotaling JM, Sorensen MD, Smith TG 3rd, Rivara FP, Wessells H, Voelzke BB. Analysis of diagnostic angiography and angioembolization in the acute management of renal trauma using a national data set. J Urol 2011;185:1316-20.

7. Loffroy R, Chevallier O, Gehin S, Midulla M, Berthod PE, Galland $\mathrm{C}$, et al. Endovascular management of arterial injuries after blunt or iatrogenic renal trauma. Quant Imaging Med Surg 2017;7:434-442.

8. Vaidya S, Tozer KR, Chen J. An overview of embolic agents. Semin Intervent Radiol 2008;25:204-15. 